\title{
A Glove for Tapping and Discrete 1D/2D Input
}

\author{
Sam A. Miller \\ NASA Langley Research Center \\ samuel.a.miller@nasa.gov
}

\begin{abstract}
This paper describes a glove with which users enter input by tapping fingertips with the thumb or by rubbing the thumb over the palmar surfaces of the middle and index fingers. The glove has been informally tested as the controller for two semi-autonomous robots in a a $3 \mathrm{D}$ simulation environment. A preliminary evaluation of the glove's performance is presented.
\end{abstract}

\section{ACM Classification Keywords}

H5.2 [Information interfaces and presentation]: User Interfaces. - Graphical user interfaces.

\section{General Terms}

Design, Human Factors

\section{Author Keywords}

Wearable, mobile, tactile, proprioception, glove-based interaction

\section{INTRODUCTION}

Glove-based interaction can support a number of different input techniques, including finger tapping, finger flexion, hand configuration, hand orientation, and gesture [2]. Familiar examples include the chording glove [9] and commercial products from Fakespace and RallyPoint. This paper describes a glove with which users enter input by tapping a fingertip with the thumb or by rubbing the thumb over the palmar surfaces of the middle and index fingers. The glove suggests a new space of design for textile-based computing [8].

As an interaction technique, rubbing has appeared in some other devices (e.g., mobile touch interfaces [6]), but not in one of its most natural settings: between the thumb and fingers. Users can specify areas, locations, and directions by exploiting the dexterity of their finger movements as well as their proprioceptive sense, their implicit knowledge of locations on the fingers that can be touched by the thumb. Moving thumb and fingers for discrete and spatial input, independent of buttons or a fixed surface, is an expressive interaction style; users describe it as fun.

Copyright 2012 Association for Computing Machinery. ACM acknowledges that this contribution was authored or co-authored by an employee, contractor, or affiliate of the U.S. Government. As such, the Government retains a nonexclusive, royalty-free right to publish or reproduce this article, or to allow others to do so, for Government purposes only. IUI'12, February 14-17, 2012, Lisbon, Portugal.

Copyright 2012 ACM 978-1-4503-1048-2/12/02...\$10.00.

\author{
Andy Smith, Sina Bahram, Robert St. Amant \\ Department of Computer Science \\ North Carolina State University \\ pmsmith4 | sbahram | stamant@ncsu.edu
}

The glove is of ordinary nonconductive fabric onto which conductive fabric, thread, and wire are mounted. The result is a set of digital and analog data channels, with current supplied by a National Instruments USB-6009 device. Figure 1 shows the glove in diagram form. For clarity, we'll refer to the digits of the hand by $\mathrm{d} 0$ through $\mathrm{d} 4$, with $\mathrm{d} 0$ being the thumb. The tips of $\mathrm{d} 0, \mathrm{~d} 3$, and $\mathrm{d} 4$ are covered in conductive fabric, and the palmar sides of $\mathrm{d} 1$ and $\mathrm{d} 2$ are covered with a grid of conductive threads, 8 on the $x$ axis (4 threads running the length of each finger) and 10 on the $y$ axis (10 threads crossing the width of each finger). The conductive threads on the $\mathrm{d} 1 / \mathrm{d} 2$ grid are woven in an over-and-under pattern such that when an $x$ thread crosses a $y$ thread, the two are separated by nonconductive fabric.

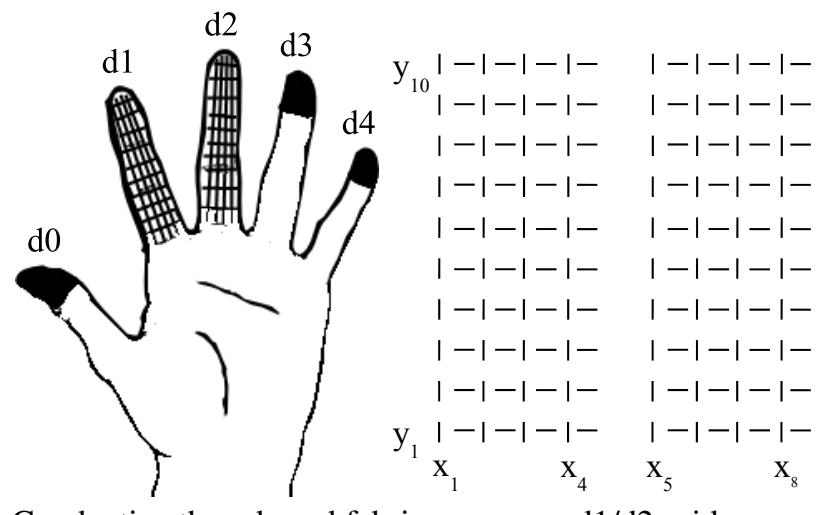

Conductive threads and fabric

$\mathrm{d} 1 / \mathrm{d} 2$ grid

Figure 1. Notional diagrams of the glove.

From an electrical circuits perspective, the glove is a set of switches, any of which can be closed by contact with $\mathrm{d} 0$. This enables the glove to detect a tap on any fingertip, which closes a switch by the thumb's contact either with conductive fabric ( $\mathrm{d} 3$ and $\mathrm{d} 4$ ) or a $y_{10}$ thread (d1 and d2). Switches can be closed in parallel, which means the glove can detect combinations of fingertip touches (i.e., $2^{4}-1=15$ ), though not all may be ergonomically feasible. The glove detects a touch to the $\mathrm{d} 1 / \mathrm{d} 2$ grid as a set of closed switches, $x_{i} \ldots x_{j}$ and $y_{m} \ldots y_{n}$, which define a rectangular boundary corresponding to the area of contact. This boundary changes as the thumb moves over the surface of the fingers. The glove has no independent clutch. Figure 2 shows the glove and the gestures it supports.

Input methods, including those for gloves, are typically categorized as being discrete or continuous, with discrete input for symbolic commands or characters, and continuous input for spatial information in one or more dimensions [1]. The 


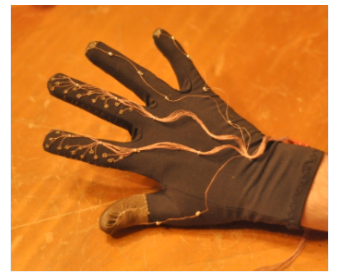

Out-of-range state

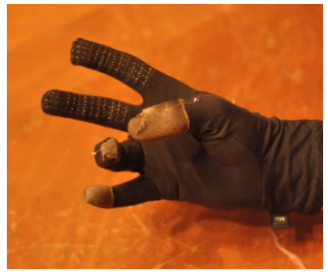

Single click

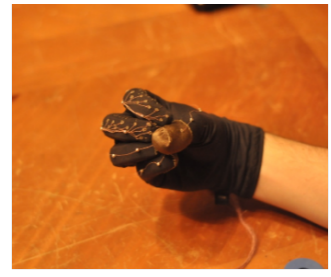

Multiple click

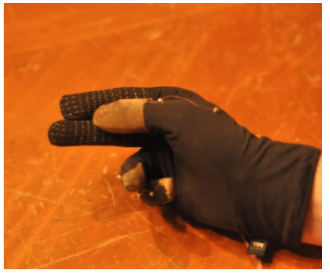

A rubbing gesture

Figure 2. The glove in use

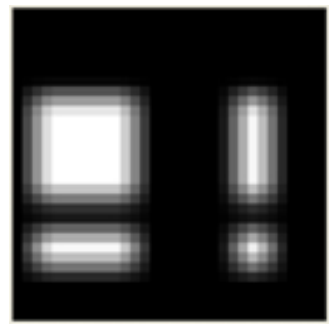

Bi-linear interpolation

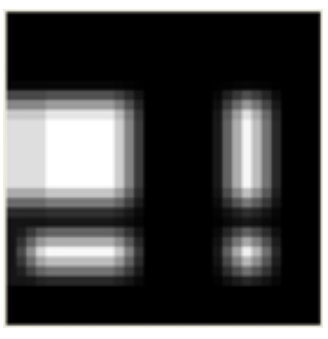

Proper close

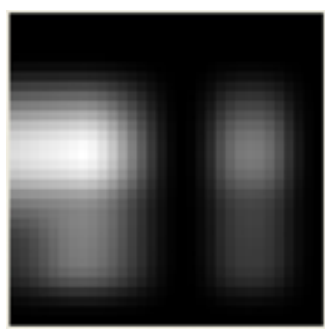

Local smoothing

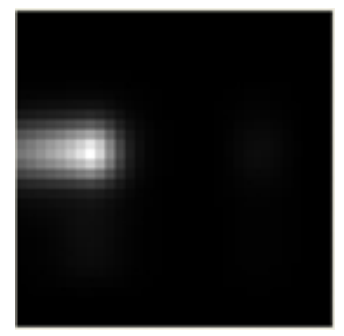

Exponential look-up

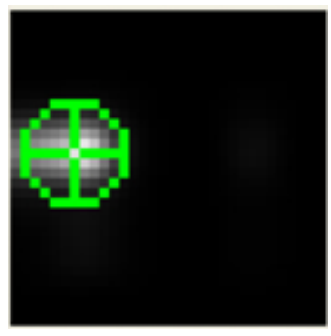

Centroid computation

Figure 3. Image processing of grid input

d1/d2 grid is comparable to a flexible touchpad attached to two fingers, supporting discretized spatial input. The body of this paper describes the glove's internal processing, a preliminary study of its performance, and an application we have built to explore its use in practice.

\section{GLOVE PROCESSING}

A simple approach to translating $\mathrm{d} 1 / \mathrm{d} 2$ signals to a location or region relies on the bounding rectangle of the closed circuits. This proves to be insufficiently robust, however. The circuits are noisy - the conductive threads are prone to fraying and shorting on each other. One or two theads may sporadically fail. The glove does not fit in the same way on hands of different sizes, which introduces further noise in the touch between thumb and fingers.

Instead, we extract information using an imaging algorithm. The algorithm treats the grid as a binary image, where each $x / y$ intersection corresponds to a pixel. The algorithm determines the location of thumb by first filtering out noise from the touch image. It then finds the energy centroid of the resulting image. Because imaging filters tend to work best on source data that is smoothly varying, the original $8 \times 10 \mathrm{im}-$ age is up-sampled using bi-linear interpolation to a $32 \times 32$ image. This introduces smooth edges onto the image. A "proper close" step removes small noise elements from image without disturbing the smoothed edges. A local-average filter with a $7 \times 7 \mathrm{kernel}$ is then applied to ramp all gray-scale values in the image, leaving brightest regions in the center of large regions. The dynamic range of the image is transformed by look-up in an exponential table to accentuate the brightest regions. Finally, the energy centroid is found for the entire image; this captures the point of contact with the $\mathrm{d} 1 / \mathrm{d} 2$ grid. This is not the only possible design for an imaging algorithm for this domain, but it is the first we discovered that worked well; machine learning techniques may improve performance. The process, from binary image to final centroid, is shown by example in Figure 3.

\section{THE GLOVE IN USE}

The initial goal for the development of the glove was to support flexible one-handed interaction with a rich virtual environment, as might be provided in an augmented reality system, while the user is walking around, looking through a head-mounted display. A simulation of such an application was developed, based on a video game engine, as shown in Figure 4. The simulation contains two semi-autonomous robots, a crawler and a flyer, that follow pre-programmed paths through a rain forest. Each robot pipes its video feed and other status information to the user. The user can take control of either machine at any time to direct its actions using buttons, menus, and navigational controls. ${ }^{1}$ The user controls the simulation with different glove gestures:

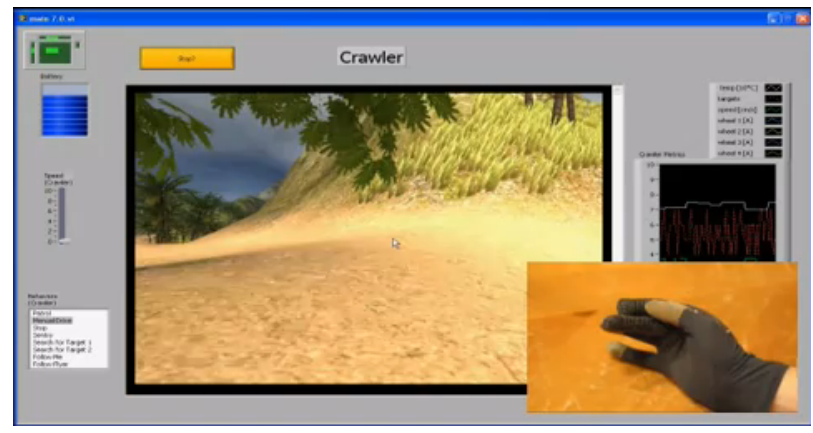

Figure 4. Control of crawler robot, in simulation.

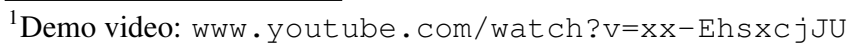


- Tapping: Some global commands are mapped to taps on fingertips: a single touch and release on one or more fingers, or repeated touches.

- Rub positioning: The robots can be steered by by rubbing d1. Menu navigation is through the same gesture, with selection mapped to a tap on $\mathrm{d} 3$. Positioning in two dimensions is also possible with rubs to the $\mathrm{d} 1 / \mathrm{d} 2$ grid; this is used in pop-up pie menus. Touch positioning, in which a touch to the grid is interpreted in absolute positioning mode, is possible but was not implemented.

- Swiping: Some context-sensitive commands are mapped to directional "swipes" on the $\mathrm{d} 1 / \mathrm{d} 2$ grid, to execute a command or change state in the interface.

In a formative evaluation, six participants used the simulation. Each session began with a demonstration of the glove's interactions. All participants could use the glove effectively within five minutes, the only consistent challenge being the difficulty of remembering the mapping of different gestures to commands. Qualitative feedback was positive: Participants found some gestures intuitive and enjoyable, in particular control of the movement of the robots by rubbing gestures. The glove generally met our expectations. It shows promise for controlling a simulation, with some commands and continuous actions naturally mapping to the glove gestures.

\section{TESTING}

We also carried out a preliminary experiment to test the glove for common interaction tasks. For brevity, we will concentrate on a single task in the experiment, summarizing the rest. The experiment involved 11 participants, all unpaid volunteers. Participants ranged in age from 21 to 33. Two were female, nine male. All were right-handed, with vision corrected to 20/20, and use computers daily. Each session lasted about 30 minutes.

The main task for the experiment was target selection in one dimension (Task 1a) or two dimensions (Task 1b). Task 1a was performed before Task $1 \mathrm{~b}$. Participants were shown a box containing a colored target region on a computer display. The spatial extent of the box was mapped in absolute terms to the $\mathrm{d} 1 / \mathrm{d} 2$ grid. In Task $1 \mathrm{a}$, the $x$ component of touch signals was ignored.

Recall that the input resolution of the glove is much lower than that of a typical display. The goal of this task was to establish a reasonable ratio for mapping input resolution to output resolution. The box was divided into an array of $n \times 1$ elements for Task 1a and $n \times n$ elements for Task $1 \mathrm{~b}$ (the resolution), and the target occupied $s \times 1$ elements for Task $1 \mathrm{a}, s \times s$ elements for Task $1 \mathrm{~b}$ (the relative target size). These divisions were not visible in the display. The specific values used for $n$ and $s$ are given in Table 1, based on our judgments about the difficulty of the task from a pilot study.

The participants carried out 24 trials for each task in a training phase. This was followed by 10 trials for each of the conditions in Table 1, with target locations generated at random. Each trial began with the participant's thumb out of contact with the other fingers. A target appeared in a random

\begin{tabular}{rrr}
\hline$s$ & $n$ & Ratio \\
\hline 2 & 9 & 0.22 \\
4 & 9 & 0.44 \\
2 & 30 & 0.06 \\
4 & 30 & 0.13 \\
8 & 30 & 0.26
\end{tabular}

Table 1. Target size $(s)$, resolution $(n)$, and target size ratio.

location, and the participant performed a Touch positioning action, causing a cursor to appear. If the cursor did not overlap the target, the participant performed a Rub positioning action to move the cursor to the target. The participant released contact to indicate completion, and the next trial began immediately. For each trial, the duration was recorded between the release event at the end of one trial and the release event of the next trial; the time for a new target to appear was considered negligible. A trial was considered successful if the release event occurred with the cursor overlapping the target.

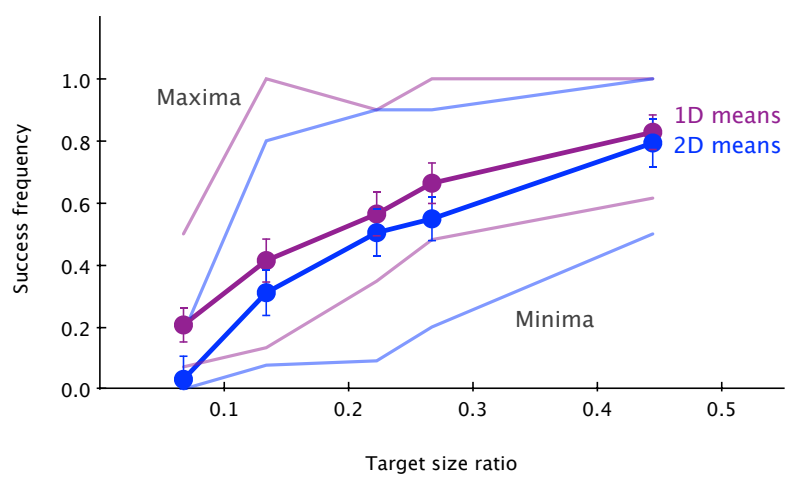

Figure 5. Task 1a/b success frequency, per target size ratio.

Some data cleaning was performed to remove outliers with durations longer than 10 seconds (less than $1 \%$ of the data for Task 1a, less than $2 \%$ of the data for Task 1b). Qualitatively, the results were largely as expected. Over all conditions, the mean duration for target selection was $1326 \mathrm{~ms}$ in Task 1a, $1562 \mathrm{~ms}$ in Task 1b. The mean duration in the fastest condition, 4/9, was $953 \mathrm{~ms}$ in Task 1a, $934 \mathrm{~ms}$ in Task 1b. The heavy lines in Figure 5 show the frequency of successful completion of a trial, which ranged from 0.21 to 0.83 across conditions for Task 1a, 0.03 to 0.79 for Task $1 \mathrm{~b}$. The fine lines in Figure 5 show minima and maxima over all participants.

Other tasks in the experiment produced the following results:

- Task 2a involved participants "playing the scales" on their fingers: touching $\mathrm{d} 1$ through $\mathrm{d} 4$, in order, and then in reverse order. After a practice phase of 24 trials, participants executed 50 trials. The mean duration per tap was $305 \mathrm{~ms}$. Task $2 b$ asked participants to follow a sequence of finger taps, shown a "map" of the digits on the display. In a practice phase, participants executed 24 trials using the interface, and then carried out 50 trials for the task, in which the finger to tap was determined randomly. The mean over all fingers was $575 \mathrm{~ms}$, with a success frequency of 0.93 . 
- Task 3 was a gesture entry task, analogous to text entry tasks [4]. The gestures were single diagonal strokes, in four possible directions, from a central point on the sensor grid on the first two fingers. Four additional longer diagonal strokes were also included, from outer points on the grid (effectively the corners of the grid). Participants carried out 24 trials to practice the gestures, with a simple recognition system providing feedback about accuracy. No feedback was provided in the trials that followed, to allow users to proceed at their own speed. Unfortunately, more than half of the participants executed the gestures so quickly (averaging 150 to $400 \mathrm{~ms}$ per gesture) that the gesture recognizer failed. The recognition rate over all participants was 0.40 . The The highest success frequency was 0.94 for one participant, whose gestures had a mean duration of $614 \mathrm{~ms}$.

We view these findings as promising but not definitive. Our formative evaluation and the high performance of the glove's developers led us to an overly optimistic experiment design. For contrast, Rosenberg and Slater's evaluation of the chording glove [9] involved ten sessions of 50 minutes each for participants to reach competence in text entry (though much of that time could be attributed to learning chords). It is clear from our experiment that the glove needs further evaluation as well as improved hardware if it is to be deployed.

We find a few bright spots in our experiment: it was possible for participants to achieve good performance using the glove, even if not reliably. The authors of this paper, with more experience, significantly outperform the best of the participants; as in other glove-based work, training plays a factor in performance. Further, our results are largely consistent with other work in mobile and glove-based interaction. For example, Parhi et al. [7] give duration results for selection of targets on small touchscreens with the thumb, in one-handed use. For targets of average size on small touch screens, selection time is between 1100 and 1200 milliseconds; errors appear in almost $30 \%$ of selections. These tasks differ from those in our Task 1, but the comparable timing and error rates suggest that interaction with the glove may reach reasonable performance in the abstract.

The simulated robot application worked much better than our experiment would suggest. Failed inputs had low costactions could be repeated - and users found moving through linear and pie menus by rub positioning, driving and flying the robots with by rub positioning, and shifting interface modes or confirming selection by tapping, all to be effective and easy to learn. This suggests that our glove has relevance for specific application domains and interaction styles, in particular those relevant to interaction when the user's hand might be out of view.

A good deal of work remains to be done. We are currently exploring alternative sensing technology for the glove; we believe that proprioception is insufficiently exploited in input devices, with only a few examples of its explicit consideration to be found in the literature (e.g. [5]). Other application areas also appear promising; for example, given the glove's expressiveness, we have solicited input from a semi-professional musician and built a glove-controlled synthesizer application. We believe that with better hardware, the glove should offer a new avenue for interaction in wearable computing.

\section{ACKNOWLEDGMENTS}

Special thanks to Chelsea Miller, an invaluable subject matter expert who sewed the prototype glove, and to Nikunj Kela and Harshmeet Singh, who contributed to early conceptual work on the use of the glove.

\section{REFERENCES}

1. S.K. Card, J.D. Mackinlay, and G.G. Robertson. The design space of input devices. In Proceedings of the ACM Conference on Human Factors in Computing Systems, pages 117-124. ACM, 1990.

2. L. Dipietro, A.M. Sabatini, and P. Dario. A survey of glove-based systems and their applications. Systems, Man, and Cybernetics, Part C: Applications and Reviews, IEEE Transactions on, 38(4):461-482, 2008.

3. J. Fredriksson, S.B. Ryen, and M. Fjeld. Real-time 3D hand-computer interaction: optimization and complexity reduction. In Proceedings of the Nordic Conference on Human-Computer Interaction, NORDCHI, pages 133-141, 2008. ACM.

4. I.S. MacKenzie and R.W. Soukoreff. Text entry for mobile computing: Models and methods, theory and practice. Human-Computer Interaction, 17(2):147-198, 2002.

5. M.R. Mine, F.P. Brooks Jr, and C.H. Sequin. Moving objects in space: exploiting proprioception in virtual-environment interaction. In Proceedings of the Annual Conference on Computer Graphics and Interactive Techniques, SIGGRAPH, pages 19-26. ACM, 1997.

6. A. Olwal, S. Feiner, and S. Heyman. Rubbing and tapping for precise and rapid selection on touch-screen displays. In Proceeding of the ACM Conference on Human Factors in Computing Systems, CHI, pages 295-304. ACM, 2008.

7. P. Parhi, A.K. Karlson, and B.B. Bederson. Target size study for one-handed thumb use on small touchscreen devices. In Proceedings of the Conference on Human-Computer Interaction with Mobile Devices and Services, pages 203-210. ACM, 2006.

8. E.R. Post and M. Orth. Smart fabric, or "wearable clothing". In Proceedings of the IEEE International Symposium on Wearable Computers, pages 167-168. IEEE, 1997.

9. R. Rosenberg and M. Slater. The chording glove: a glove-based text input device. IEEE Transactions on Systems, Man, and Cybernetics, Part C: Applications and Reviews, 29(2):186-191, 1999. 